\title{
Modulation Schemes Effect on the Driver Efficiency and the Global VLC Transmitter Energy Consumption
}

\author{
Guillermo del Campo-Jimenez and Franciso Jose \\ Lopez-Hernandez \\ CeDInt-UPM \\ Madrid, Spain \\ gcampo@cedint.upm.es, \\ francisco.lopez.hernandez@upm.es
}

\author{
Rafael Perez-Jimenez \\ IDETIC-ULPGC \\ Las Palmas de Gran Canaria, Spain \\ rafel.perez@ulpgc.es
}

\begin{abstract}
Visible Light Communications (VLC) make the most of the fast switching times of LEDs to transmit information. High transmission rates have been achieved with different modulation schemes as CSK or OFDM. However, the main asset of LED lamps, which is energy efficiency, has been overlooked. In this paper we analye how the modulation schemes affect the energy efficiency of LED lamps. We compare the effect of switched waveform (OOK, VPPM) and continuous waveform (OFDM) modulation schemes. Switched waveform signals present energy efficiency results between $15 \%$ and $30 \%$ better than continuous waveform signals.
\end{abstract}

Keywords—VLC; Driver; Energy Efficency

\section{INTRODUCTION}

Over the last decade, traditional incandescent lamps have been progressively replaced by white light emitting diodes (WLED). This replacement is motivated by LED long lifetime and low heat generation, but mainly because of LED high energy efficiency. A commercial WLED is currently able to emit more than $180 \mathrm{~lm} / \mathrm{W}$ [1,2], although the overall efficiency falls to $75-100 \mathrm{~lm} / \mathrm{W}$ including the power supply. In order to assure a high electrical-optical conversion efficiency, a welladapted power supply or driver is required. LED drivers should supply a constant current or voltage to the LED (or LED array) and are usually based on switched voltage sources, as they require efficiencies of more than $90 \%$ [3]. Dimming capability, which is necessary in illumination, may be accomplished with a pulse width modulation (PWM) modulated bias current using a switching metal oxide semiconductor (MOS) transistor. PWM signal controls the emitted light power so as to provide a desired mean value while its switching frequency should be high enough to avoid flickering effects.

Visible light communication (VLC) makes use of the LED short switching times to transmit information. The VLC IEEE 802.15.7 standard [4] defines three physical layers (PHY) and their related modulation schemes depending on the channel interface. PHY1 is intended for low data rate communications ( $<267 \mathrm{Kbit} / \mathrm{s}$ ), indicating the use of On-Off keying (OOK) and variable pulse position modulation (VPPM). PHY2 and PHY3 are designed for indoor applications with moderate and high data rates $(<96 \mathrm{Mbit} / \mathrm{s})$, using OOK and VPPM and color shift keying (CSK) respectively. CSK can be used when white illumination is produced through the combination of red, green and blue (RGB) LEDs. Dimming can be achieved either by adding an extension to the transmission frame to adjust the average bias level (OOK, CSK) or by changing the pulse duty cycle (VPPM).

For OOK and VPPM modulation schemes, the transmission rate is restricted by the switching time of a WLED, determined by its phosphor layer, which is about several hundred nanoseconds, i.e. a bandwidth of a few megahertz (typically 2$3 \mathrm{MHz}$ ) [5]. In order to overcome this limitation, the phosphor delay can be removed at the receiver using a blue filter, which increases the bandwidth up to $20 \mathrm{MHz}$ [6]. A $175 \mathrm{MHz}$ bandwidth could be reached using high-frequency driver circuits [7-9]. On the other hand, when using RGB sources, the bandwidth can be increased by enabling the modulation of each color independently, known as wavelength-division multiplexing (WDM) or CSK [10-12].

High spectrally efficient techniques such as orthogonal frequency-division multiplexing (OFDM) and discrete multitone (DMT) can be used to further increase the transmission bandwidth. Due to the available signal power of VLC systems, the tradeoff between the data rate and SNR can be used. OFDM is based on the generation of a multicarrier signal, whereas each sub-carrier is independently modulated (M-QAM, MPSK) to create several parallel communications channels. Considering illumination is a unipolar signal, the traditional RF bipolar OFDM has to be adjusted. Optical OFDM can be achieved either by adding a dc-bias signal, known as DC biased optical OFDM (DCO-OFDM) [13] or by removing all the negative values at the original bipolar modulating signal, known as asymmetrically clipped OFDM 
(ACO-OFDM) [14]. ACO-OFDM is more efficient for low symbol constellations, while DCO-OFDM produces a better performance for larger constellations. Asymmetrically clipped DC biased optical OFDM (ADO-OFDM) combines both techniques to achieve greater optical power efficiency [15]. The Short-Range Optical Wireless Communications Task Group (TG 7r1) is preparing the revision of the IEEE 802.15.7r1 standard [16] and includes OFDM for high data rate communications. IEEE $802.15 .7 \mathrm{r} 1$ proposes the use of OFDM in combination with adaptive mechanisms to adjust the system to channel variations brought about by multipath effects (e.g. wall reflections) and receiver mobility [17-19]. Many theoretical works and experiments show the applicability of OFDM and DMT schemes, reaching data rates over 1 Gbps [20-23].

The most part of research efforts have focused on increasing the transmission rate, achieving remarkable results. However, the main function of LED lamps, which is lighting, and their most relevant advantage over traditional lamps, which is energy efficiency, have been overlooked.

In this work, we study how the VLC modulation schemes affect the overall energy efficiency of an LED lamp. To illustrate how the signal waveform affects the energy efficiency of the lamp, first we analyse basic switched and sinusoidal signals. Afterwards, we compare the effect of three modulation techniques: OOK, VPPM and OFDM. Results show that when using continuous waveform (CW) signals instead of switched waveform (SW) signals, the energy efficiency of LED lamps becomes a significant issue.

\section{THEORICAL MODEL}

To clarify the effect of the signal waveform on the energy efficiency of an LED lamp, we consider a simplified model of an LED modulating driver circuit (see Fig. 1). For this work a single LED has been considered, though results with LED arrays are similar according to our simulations and experiments. An efficient power supply produces the bias voltage $\mathrm{V}_{\mathrm{DD}}$. This voltage is applied to the LED (or LED array) and the current $\left(\mathrm{I}_{\mathrm{LED}}\right)$ is controlled by the driver.

An LED driver, in its basic setup, is formed by a MOS transistor, which controls the current applied to the LED. How this control is achieved while securing an optimal energy performance, has been the subject of many research works [2430]. The best method for driving an LED for illumination is using a current constant source configuration. However, due to

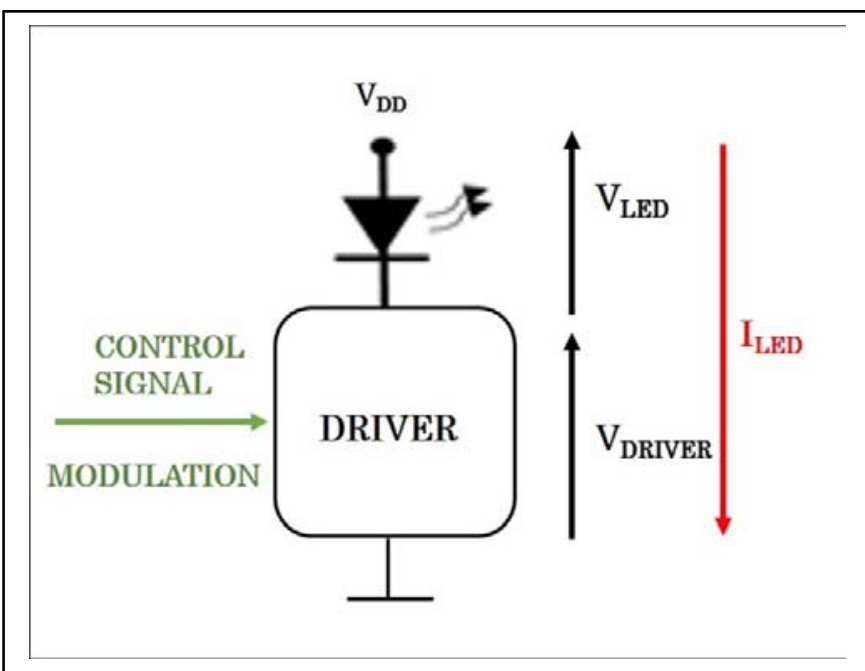

Fig. 1. Model of an LED modulating circuit. its high impedance, it is not fast enough for the modulation function. In this work, we use a voltage constant configuration, where the MOS incorporates both VLC functions (control and modulation) in a single device. Control and modulation may be implemented using separated devices, e.g. a bias-T [31,32]. Concerning the scope of this work, i.e. the energy efficiency of drivers, would be the same for a single and two devices configuration, where it would be necessary to add the power dissipation from both the illumination and the modulation devices.

We define $\eta$, the ratio between the power dissipated by the LED and the total dissipated power, in order to analyse the energy efficiency of the driver:

$$
\eta=\frac{P_{L E D}}{P_{T O T A L}}
$$

Equation (1) defines the amount of power wasted in the driver, reducing the lamp's efficiency. As $\mathrm{I}_{\mathrm{LED}}=\mathrm{I}_{\mathrm{DRIVER}}, \eta$ can be calculated as:

$$
\eta=\frac{<v_{L E D}(t) \times i_{L E D}(t)>}{<v_{L E D}(t) \times i_{L E D}(t)>+<v_{\text {Driver }}(t) \times i_{L E D}(t)>}
$$

Equation (2) shows that $\eta$ depends on the current waveform ( $\left.\mathrm{I}_{\mathrm{LED}}\right)$ and both LED and MOS voltages $\left(\mathrm{V}_{\mathrm{LED}}\right.$ and $\left.\mathrm{V}_{\text {DRIVER }}\right)$. Both instantaneous current and voltages depend on the signal waveform. The energy efficiency ratio $\eta$ can vary from between 0 (completely inefficient) to 1 (ideally efficient). $V_{\text {LED }}$ and $\mathrm{V}_{\text {DRIVER }}$ are determined by the LED and MOS I-V characteristic curves including transition effects. The I-V curves of the used commercial LED (HP803WW) and MOS (MGSF1N03LT1G) have been measured experimentally for this study.

For a first analysis, we have considered square and sinusoidal signals, as examples of SW and CW signals. In Fig. 2 we represent the joint I-V curves of the LED and MOS. For the correct comparison of the signals, we establish the next two

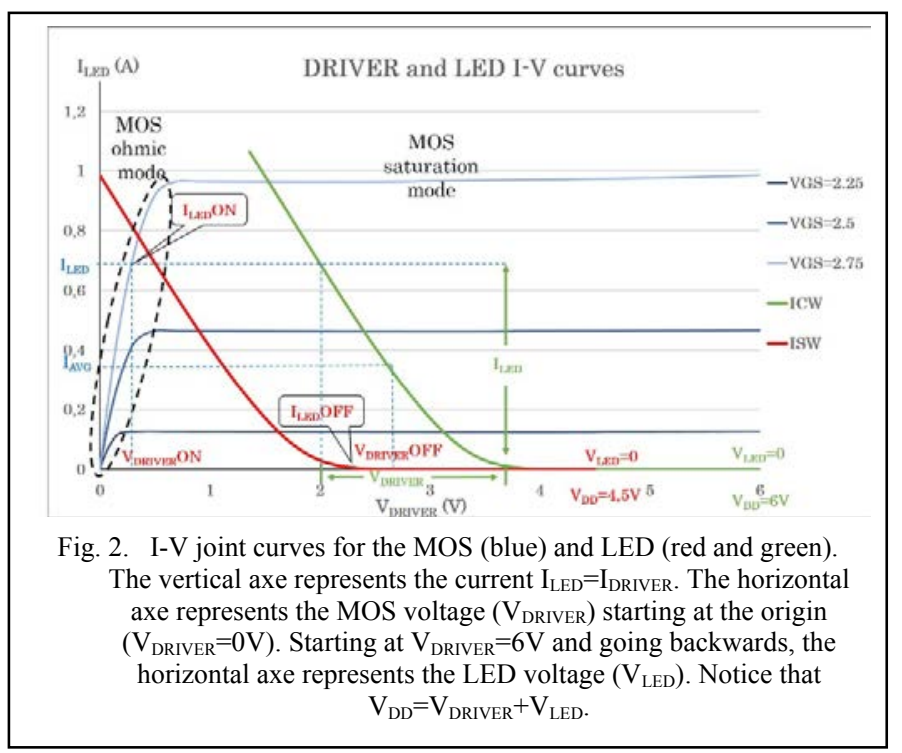


constraints: current average $\left(\mathrm{I}_{\mathrm{AVG}}\right)$, which sets the level of illumination and must be the same for both $\mathrm{SW}$ and $\mathrm{CW}$; and current peak ( $\left.\mathrm{I}_{\mathrm{MAX}}\right)$, which is limited by the LED characteristics $\left(\mathrm{I}_{\mathrm{MAX}}=1 \mathrm{~A}\right)$.

The MOS curves for different gate voltages $\left(\mathrm{V}_{\mathrm{GS}}\right)$ are printed in blue. The red and green lines represent the I-V curves of an LED, drawn from the right hand side, i.e. $\mathrm{V}_{\mathrm{LED}}=0 \mathrm{~V}$ for $\mathrm{V}_{\mathrm{DD}}=4.5 \mathrm{~V}$ and $6 \mathrm{~V}$ respectively.

The red line corresponds to a square (SW) signal, where the driver switches between the low state (OFF) and the high state $(\mathrm{ON})$. For these $\mathrm{V}_{\mathrm{GS}}$ values, the MOS changes between the cutoff mode $\left(\mathrm{V}_{\mathrm{GS}}<\mathrm{V}_{\mathrm{th}}\right)$, where the MOS acts as an open circuit and there is no current; and the ohmic mode $\left(V_{G S}>V_{\text {th }}\right.$ and $\mathrm{V}_{\text {DRIVER }}<\left(\mathrm{V}_{\mathrm{GS}}-\mathrm{V}_{\text {th }}\right)$ ), where the MOS acts as a resistor controlled by the gate voltage $\left(\mathrm{V}_{\mathrm{GS}}\right)$ relative to both the source and drain voltages, i.e. it depends on $\mathrm{V}_{\text {DRIVER }}$. This switching is efficient because there is no power consumption in the cutoff mode, and at the ohmic mode, $\mathrm{V}_{\text {DRIVER }}$ is very low, so the power dissipated at the driver is low. The level of illumination $\left(\mathrm{I}_{\mathrm{AVG}}\right)$ can be dimmed by varying the higher level of the SW signal or even $\mathrm{V}_{\mathrm{DD}}$, although the optimal methods change the duty cycle of the signal or add an extension to adjust the level of illumination.

On the other hand, to obtain a CW signal at the LED (green line in Fig. 2), the driver must be modulated with a sinusoidal signal. The level of illumination $\left(\mathrm{I}_{\mathrm{AVG}}\right)$ is determined by the signal offset. To avoid signal distortion or clipping, the MOS must be at the saturation or conduction mode $\left(\mathrm{V}_{\mathrm{GS}}>\mathrm{V}_{\text {th }}\right.$ and $\left.\mathrm{V}_{\text {DRIVER }}>\left(\mathrm{V}_{\mathrm{GS}}-\mathrm{V}_{\mathrm{th}}\right)\right)$, where the current depends directly on $\mathrm{V}_{\mathrm{GS}}$, which is independent of $\mathrm{V}_{\text {DRIVER}}$. In this mode, power dissipation at the driver is increased since there is always current $\left(\mathrm{I}_{\mathrm{LED}}>0\right)$ and $\mathrm{V}_{\text {DRIVER }}$ is higher than in the ohmic mode.

Fig. 3 shows the effect of the signal waveform on the power dissipation over time, specifically during a signal period. Red colored lines represent a SW (square) signal. When the driver signal $\left(\mathrm{V}_{\mathrm{GS}}\right)$ is set to a low value, it causes the MOS to be at the cutoff mode, where the voltage at the driver $\mathrm{V}_{\text {DRIVER }}$ is high, but there is not current $\left(\mathrm{I}_{\mathrm{LED}}=0\right)$, and consequently there is not

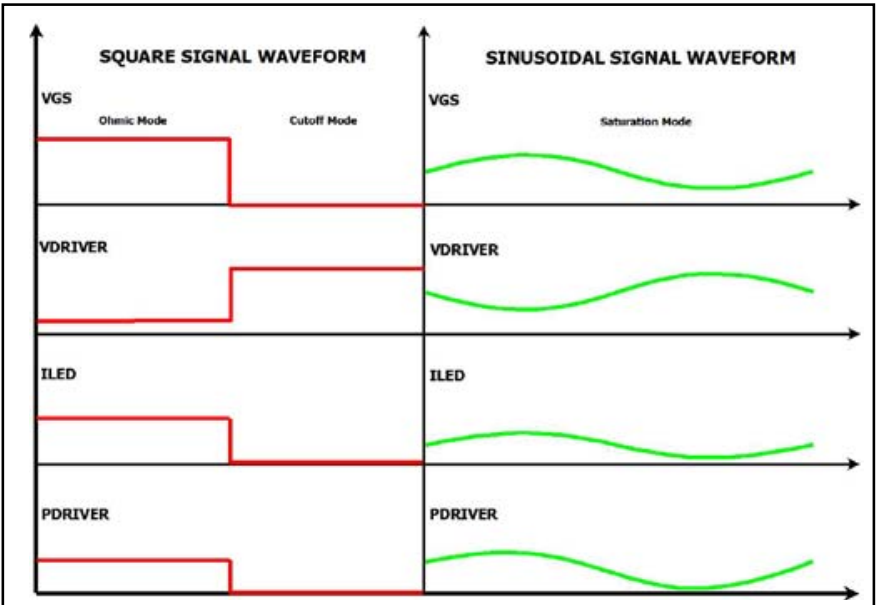

Fig. 3. Driver signals during a period time: SW (square) signal in red and $\mathrm{CW}$ (sinusoidal) in green). power dissipation $\left(\mathrm{P}_{\mathrm{DRIVER}}=0\right)$. On the other hand, when $\mathrm{V}_{\mathrm{GS}}$ is set to value so the MOS is in the ohmic mode, there is $\mathrm{I}_{\mathrm{LED}}$ and $\mathrm{V}_{\text {DRIVER }}$ is low, resulting in a low $\mathrm{P}_{\text {DRIVER. Green colored lines }}$ represent a $\mathrm{CW}$ (sinusoidal) signal. In order to avoid clipping and signal distortion, $\mathrm{V}_{\mathrm{GS}}$ values move within a range where the MOS is at saturation mode. As a result, $\mathrm{P}_{\text {DRIVER }}$ is higher, since there always happen to exist simultaneously both $V_{\text {DRIVER }}$ and $\mathrm{I}_{\mathrm{LED}}$.

\section{EXPERIMENTAL RESULTS}

We have modeled and simulated an LED driver circuit and calculated the driver efficiency ratio $\eta$ for different illumination values $\left(\mathrm{I}_{\mathrm{AVG}}\right)$. Table I shows the efficiency ratio for the square signal. The signal modulating the driver is set to an On-Off 200 $\mathrm{KHz}$ switching with a constant high value. The level of illumination is determined by the duty cycle (dc) and the supply voltage $\mathrm{V}_{\mathrm{DD}}$. Generally, $\mathrm{V}_{\mathrm{DD}}$ will have a constant value, though it could be regulated to compensate the thermal effects. As can be seen in Table 1 , above $\mathrm{V}_{\mathrm{DD}}=5.3 \mathrm{~V}$, the driver (MOS) changes into the saturation mode and is no longer efficient ( $\mathrm{I}_{\mathrm{AVG}}$ does not further increase).

Table II shows the efficiency ratio $\eta$ calculation for the sinusoidal signal. The driver modulating signal is a $200 \mathrm{KHz}$ sinusoidal wave with an offset, which determines the level of illumination, and an amplitude, which is limited to avoid clipping. It can be seen how $\eta$ improves when $V_{D D}$ is reduced down to $5.4 \mathrm{~V}$, the limit to avoid distorting the optical signal for the given amplitude. Lower $\mathrm{V}_{\mathrm{DD}}$ could be used by decreasing the signal amplitude, although it would mean a critical drop in the optical signal amplitude and consequently the SNR. It is also important to point out the $\eta$ deterioration for low illumination signals (low $\mathrm{I}_{\mathrm{AVG}}$ ).

Looking at the energy efficiency ratio $\eta$ of the two waveforms, we can see that the square signal presents much better values $(\geq 0.88)$ than the sinusoidal signal $(\leq 0.73)$. The difference increases for low illumination values $(\geq 0.87)$ versus $(\leq 0.58)$.

TABLE I. EFFICIENCY RATIO H FOR A SQUARE (SWITCHED) SIGNAL

\begin{tabular}{|c|c|c|c|c|c|c|}
\hline \multirow{2}{*}{$\begin{array}{l}\mathbf{V}_{\text {DD }} \\
(\mathbf{V})\end{array}$} & \multicolumn{2}{|c|}{$\mathbf{d c}=\mathbf{2 5 \%}$} & \multicolumn{2}{c|}{$\mathbf{d c}=\mathbf{5 0} \%$} & \multicolumn{2}{c|}{$\mathbf{d c}=\mathbf{7 5 \%}$} \\
\cline { 2 - 7 } & $\boldsymbol{I}_{\boldsymbol{A} \boldsymbol{V} \boldsymbol{G}}(\boldsymbol{A})$ & $\boldsymbol{\eta}$ & $\boldsymbol{I}_{\boldsymbol{A} \boldsymbol{V} \boldsymbol{G}}(\boldsymbol{A})$ & $\boldsymbol{\eta}$ & $\boldsymbol{I}_{\boldsymbol{A} \boldsymbol{V} \boldsymbol{G}}(\boldsymbol{A})$ & $\boldsymbol{\eta}$ \\
\hline $\mathbf{4}$ & 0.099 & 0.938 & 0.199 & 0.946 & 0.299 & 0.949 \\
\hline $\mathbf{4 . 2 5}$ & 0.126 & 0.927 & 0.252 & 0.935 & 0.379 & 0.938 \\
\hline $\mathbf{4 . 5}$ & 0.153 & 0.916 & 0.306 & 0.924 & 0.46 & 0.926 \\
\hline $\mathbf{4 . 7 5}$ & 0.18 & 0.903 & 0.36 & 0.911 & 0.54 & 0.914 \\
\hline $\mathbf{5}$ & 0.205 & 0.89 & 0.410 & 0.897 & 0.615 & 0.900 \\
\hline $\mathbf{5 . 2 5}$ & 0.227 & 0.873 & 0.454 & 0.881 & 0.682 & 0.883 \\
\hline$\geq \mathbf{5 . 3}$ & \multicolumn{6}{|c|}{ Driver (MOS) in saturation mode $\left(\mathrm{I}_{\mathrm{AVG}}\right.$ does not increase) } \\
\hline
\end{tabular}

TABLE II. EFFICIENCY RATIO H FOR A SINUSOIDAL SIGNAL

\begin{tabular}{|c|c|c|c|c|c|c|}
\hline \multirow{2}{*}{$\begin{array}{l}V_{\text {DD }} \\
(\mathbf{V})\end{array}$} & \multicolumn{2}{|c|}{ offset $=\mathbf{2 5} \%$} & \multicolumn{2}{c|}{ offset $=\mathbf{5 0} \%$} & \multicolumn{2}{c|}{ offset $=\mathbf{7 5 \%}$} \\
\cline { 2 - 7 } & $\boldsymbol{I}_{\boldsymbol{A} \boldsymbol{V} \boldsymbol{G}}(\boldsymbol{A})$ & $\boldsymbol{\eta}$ & $\boldsymbol{I}_{\boldsymbol{A} \boldsymbol{V} \boldsymbol{G}}(\boldsymbol{A})$ & $\boldsymbol{\eta}$ & $\boldsymbol{I}_{\boldsymbol{A} \boldsymbol{V} \boldsymbol{G}}(\boldsymbol{A})$ & $\boldsymbol{\eta}$ \\
\hline$\leq \mathbf{5 . 4}$ & \multicolumn{5}{|c|}{ Driver (MOS) in ohmic mode, clipping. } \\
\hline $\mathbf{5 . 5}$ & 0.242 & 0.582 & 0.481 & 0.666 & 0.721 & 0.732 \\
\hline $\mathbf{5 . 7 5}$ & 0.242 & 0.551 & 0.481 & 0.648 & 0.721 & 0.708 \\
\hline $\mathbf{6}$ & 0.242 & 0.525 & 0.481 & 0.631 & 0.721 & 0.686 \\
\hline
\end{tabular}


After evaluating the effect of basic $\mathrm{SW}$ and $\mathrm{CW}$ signals, for the next step we consider actual VLC modulation signals: OOK, VPPM and OFDM. We study the effect for the transmission of a 128 bit data packet. As for the basic waveforms, the constraints are the level of illumination and current peaks.

First, we study the effect of an OOK modulation signal, with the parameters established by the IEEE 802.15.7 standard: $200 \mathrm{KHz}$ clock frequency, Manchester encoded. Table III shows the results. As expected, we can see that the OOK signal presents a similar behavior (and $\eta$ ) compared to the basic SW (square) signal.

Secondly, we analye the effect of a VPPM signal. We adjust it to the parameters of the IEEE 802.15.7 standard while maintaining the illumination level and the transmission rate: $400 \mathrm{KHz}$ clock frequency, 4B6B encoded. Table IV shows the results. VPPM and OOK results are very similar, being the small differences caused by the change of clock frequency.

Finally, we study the effect of an OFDM signal. We consider a signal with 4 sub-carriers. Each sub-carrier uses a binary phase shift keying (BPSK) modulation. In order to match the OOK transmission rate, we use a $50 \mathrm{KHz}$ BPSK modulated sinusoidal signal. Table $\mathrm{V}$ shows the energy efficiency ratio $\eta$ results for the 128 bit data packet transmission.

TABLE III. EFFICIENCY RATIO H FOR AN OOK SIGNAL

\begin{tabular}{|c|c|c|c|c|c|c|}
\hline \multirow{2}{*}{$\begin{array}{l}V_{\text {DD }} \\
(V)\end{array}$} & \multicolumn{2}{|c|}{ dc $=25 \%$} & \multicolumn{2}{|c|}{ dc $=50 \%$} & \multicolumn{2}{|c|}{$d c=75 \%$} \\
\hline & $I_{A V G}(A)$ & $\eta$ & $I_{A V G}(A)$ & $\eta$ & $I_{A V G}(A)$ & $\eta$ \\
\hline 4 & 0.100 & 0.930 & 0.201 & 0.943 & 0.301 & 0.947 \\
\hline 4.25 & 0.127 & 0.919 & 0.254 & 0.932 & 0.381 & 0.936 \\
\hline 4.5 & 0.154 & 0.908 & 0.308 & 0.920 & 0.463 & 0.924 \\
\hline 4.75 & 0.181 & 0.895 & 0.362 & 0.908 & 0.543 & 0.912 \\
\hline 5 & 0.206 & 0.882 & 0.422 & 0.894 & 0.619 & 0.898 \\
\hline 5.25 & 0.229 & 0.865 & 0.457 & 0.877 & 0.686 & 0.881 \\
\hline$\geq 5.3$ & \multicolumn{6}{|c|}{ Driver (MOS) in saturation mode $\left(\mathrm{I}_{\mathrm{AVG}}\right.$ does not increase $)$} \\
\hline
\end{tabular}

TABLE IV. EFFICIENCY RATIO H FOR A VPPM SIGNAL

\begin{tabular}{|c|c|c|c|c|c|c|}
\hline \multirow{2}{*}{$\begin{array}{l}V_{\text {DD }} \\
(\mathbf{V})\end{array}$} & \multicolumn{2}{|c|}{$\mathbf{d c}=\mathbf{2 5 \%}$} & \multicolumn{2}{c|}{$\mathbf{d c}=\mathbf{5 0} \%$} & \multicolumn{2}{c|}{$\mathbf{d c}=\mathbf{7 5 \%}$} \\
\cline { 2 - 7 } & $\boldsymbol{I}_{\boldsymbol{A V G}}(\boldsymbol{A})$ & $\boldsymbol{\eta}$ & $\boldsymbol{I}_{\boldsymbol{A} \boldsymbol{V} \boldsymbol{G}}(\boldsymbol{A})$ & $\boldsymbol{\eta}$ & $\boldsymbol{I}_{\boldsymbol{A V G}}(\boldsymbol{A})$ & $\boldsymbol{\eta}$ \\
\hline $\mathbf{4}$ & 0.100 & 0.928 & 0.201 & 0.942 & 0.301 & 0.949 \\
\hline $\mathbf{4 . 2 5}$ & 0.127 & 0.917 & 0.254 & 0.931 & 0.381 & 0.937 \\
\hline $\mathbf{4 . 5}$ & 0.154 & 0.905 & 0.308 & 0.919 & 0.462 & 0.926 \\
\hline $\mathbf{4 . 7 5}$ & 0.180 & 0.893 & 0.362 & 0.907 & 0.542 & 0.913 \\
\hline $\mathbf{5}$ & 0.205 & 0.879 & 0.413 & 0.893 & 0.618 & 0.899 \\
\hline $\mathbf{5 . 2 5}$ & 0.228 & 0.863 & 0.457 & 0.876 & 0.685 & 0.883 \\
\hline$\geq \mathbf{5 . 3}$ & \multicolumn{6}{|c|}{ Driver (MOS) in saturation mode $\left(\mathrm{I}_{\mathrm{AVG}}\right.$ does not increase) } \\
\hline
\end{tabular}

TABLE V. EFFICIENCY RATIO H FOR AN OFDM SIGNAL

\begin{tabular}{|c|c|c|c|c|c|c|}
\hline \multirow{2}{*}{$\begin{array}{l}V_{\text {DD }} \\
(\mathbf{V})\end{array}$} & \multicolumn{2}{|c|}{ offset $=25 \%$} & \multicolumn{2}{|c|}{ offset $=50 \%$} & \multicolumn{2}{|c|}{ offset $=75 \%$} \\
\hline & $I_{A V G}(A)$ & $\eta$ & $I_{A V G}(A)$ & $\eta$ & $I_{A V G}(A)$ & $\eta$ \\
\hline$\leq 5.4$ & \multicolumn{6}{|c|}{ Driver (MOS) in ohmic mode, clipping. } \\
\hline 5.5 & 0.248 & 0.644 & 0.489 & 0.727 & 0.714 & 0.795 \\
\hline 5.75 & 0.248 & 0.6 & 0.489 & 0.696 & 0.714 & 0.760 \\
\hline 6 & 0.248 & 0.591 & 0.489 & 0.667 & 0.714 & 0.727 \\
\hline
\end{tabular}

Although the behavior is similar to that achieved for the sinusoidal signal, $\eta$ presents improved values (around $6 \%$ better). This performance is caused by the multi-carrier nature of the OFDM signal which leads to an average lower amplitude compared to the simple sinusoidal waveform, i.e. higher optical power efficiency. Energy efficiency could be improved by further reducing the signal amplitude, giving rise to SNR reduction and transmission degradation.

Fig. 4 summarizes the energy efficiency ratio $\eta$ results: (a) for SW signals with the driver in the ohmic mode; (b) for CW signals with the driver in the saturation mode. Notice the different $\mathrm{X}$-axis ranges in both figures.

There is a clear difference between SW signals (square, OOK and VPPM) versus CW signals (sinusoidal and OFDM). This difference is brought about by the waveform shape and the driver (MOS) working mode dependency. As we can see in Fig. 4(a), SW signals allow the driver to be in the ohmic mode, keeping a high efficiency. By adjusting the power supply $V_{D D}$ to the optimal value ( $4 \mathrm{~V}$ for this circuit), $\eta$ can reach values of close to 0.95 . As it can be observed, driver energy efficiency for SW signals is virtually independent of the level of illumination, which is determined by the duty cycle. On the

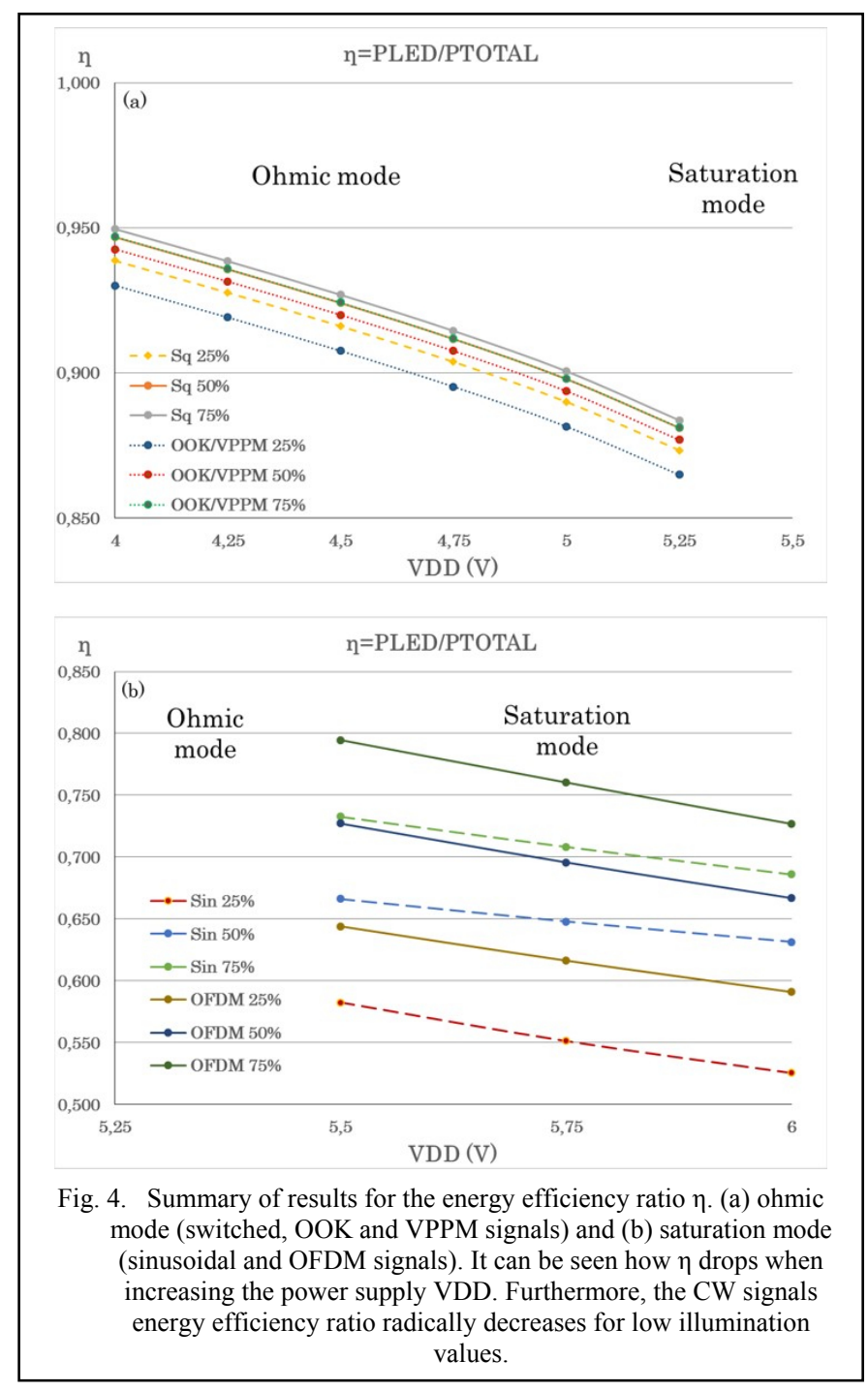


other hand (see Fig. 4(b)), the CW signals force the MOS to be in the saturation mode to avoid clipping, where the efficiency drops significantly. By regulating the power supply $\mathrm{V}_{\mathrm{DD}}$ to the lower value without signal distortion (5.5V for this circuit), $\eta$ cannot reach the 0.8 mark, for the best case, which is the high illumination level OFDM signal. Since for $\mathrm{CW}$ signals $\eta$ is illumination-dependent, it drastically degenerates for low levels of illumination.

In summary, the energy efficiency ratio $\eta$ is higher for SW signals than for CW signals: $\eta \leq 0.95$ vs $\eta \geq 0.795$ for high levels of illumination; and $\eta \geq 0.865$ vs $\eta \leq 0.644$ for low levels of illumination.

VLC emitters are LED lamps, generally formed by and an array of high power LEDs, resulting in a high emitted optical power. Therefore, the SNR, and consequently the Bit Error Rate (BER) and the Channel Capacity (C), depend directly on the optical signal amplitude. For SW signals, the signals amplitude is constant and equals to $\mathrm{I}_{\mathrm{MAX}}$. For $\mathrm{CW}$, the amplitude is affected by dimming: it is $\mathrm{I}_{\mathrm{MAX}}$ for the $50 \%$ illumination level signal, but is limited to $2\left(\mathrm{I}_{\mathrm{MAX}}-\mathrm{I}_{\mathrm{AVG}}\right)$ for dimming $>50 \%$ and $2 \mathrm{I}_{\mathrm{AVG}}$ for dimming $<50 \%$. Reducing signal amplitude below these values could improve the energy efficiency of $\mathrm{CW}$ signals (enabling $\mathrm{V}_{\mathrm{DD}}$ reduction without distortion). However, amplitude reduction would cause SNR degradation, minimizing the spectral efficiency.

For SW signals the SNR remains almost constant for different energy efficiency ratios, while for CW signals, SNR is inversely proportional to $\eta$. In order to compare the tradeoff between the energy efficiency and the achievable data rates of the modulation techniques, schemes with comparable data rates, as CSK and OFDM should be analysed. This analysis will be presented in future works.

\section{CONCLUSIONS}

In conclusion, this work shows, that the waveform shape strongly influences the overall energy efficiency of an LED lamp. SW signal-based modulations (such as OOK or VPPM) have a high energy performance $(0.86 \leq \eta \leq 0.95)$, while $\mathrm{CW}$ signal-based modulations (such as OFDM) reduce energy efficiency $(0.59 \leq \eta \leq 0.79)$, mainly constrained by the signal amplitude and the level of illumination.

It is important to remember that the primary lamp function is lighting, and that the main reason why LED lamps are replacing traditional lighting sources is their energy efficiency. Therefore, when using LED lamps as VLC transmission devices, a balance between energy efficiency and binary rates should be achieved.

We think this work should help to raise awareness on the energy efficiency of VLC drivers and boost research community to find solutions to this issue.

The effect on the emitter efficiency of the other modulation scheme proposed in the IEEE 802.15.7 standard (CSK) is currently being researched and will be presented in future works. High energy efficiency results are expected as CSK use signals with similar characteristics to OOK and VPPM, i.e. SW signals.

\section{ACKNOWLEDGMENT}

This work was supported in part by the Spanish Government, MINECO National Research Plan (ARIES Project Ref. TEC2013-47682-C2-1).

\section{REFERENCES}

[1] S. Nizamoglu, G. Zengin and H. V. Demir, "Color-converting combinations of nanocrystal emitters for warm-white light generation with high color rendering index", Appl. Phys. Lett. 92, 031102 (2008).

[2] "New XLamp® XP-G2 LEDs Deliver 20 Percent Efficiency Increase in the XP Footprint", Cree News \& Events, http://www.cree.com/newsand-events/cree-news/press-releases/2012/july/xpg2-intro (July 10, 2012).

[3] H. Chiu, Y. Lo, J. Chen, S. Cheng, C. Lin and S. Mou, "A High.Efficiency Dimmable LED Driver for Low-Power Lighting Applications", IEEE Trans. Industrial. Electr. 57, 2-735 (2010).

[4] "IEEE Standard for Local and Metropolitan Area Networks--Part 15.7: Short-Range Wireless Optical Communication Using Visible Light", (2011).

[5] T. Komine and M. Nakagawa, "Fundamental analysis for visible light communication system using LED lightings", IEEE Trans. Consum. Electron. 50, 100 (2004).

[6] J. Grubor, K.-D. Langer, S. C. J. Lee, T. Koonen, and J. W. Walewski, "Wireless high-speed data transmission with phosphorescent white-light LEDs", in Proceedings of 33rd Eur. Conf. Exhib. Opt. Commun. (2007).

[7] L. Grobe, A. Paraskevopoulos, J. Hilt, D. Schulz, F. Lassak, F. Hartlieb, C. Kottke, V. Jungnickel, and K.-D. Langer, "High-Speed Visible Light Communication Systems”, IEEE Comm. Mag. 51, 1260 (2013).

[8] H. Li, X. Chen, J. Guo, Z. Gao, and H. Chen, “An Analog Modulator for $460 \mathrm{MB} / \mathrm{S}$ Visible Light Data Transmission Based on OOK-NRS Modulation", IEEE Wirel. Comm. 22, 2-68 (2015).

[9] J. Vucic, C. Kottke, S. Nerreter, K. Habel, A. Buttner, K.-D. Langer and J.W. Walewski, "125 Mbit/s over $5 \mathrm{~m}$ wireless distance by use of OOKModulated phosphorescent white LEDs", in Proceedings of European Conference on Optical Communication (2009).

[10] J.M. Luna-Rivera, R. Perez-Jimenez, J. Rabadan-Borjes, J. Rufo-Torres, V. Guerra, and C. Suarez-Rodriguez, "CSK scheme for indoor visible light communications”, Optic Express 22, 024256 (2014).

[11] R. Singh, T. O'Farrell, J.P.R. David, "An Enhanced Color Shift Keying Modulation Scheme for High-Speed Wireless Visible", J. Lightwave Tech. 32, 142582 (2014).

[12] Y. Wu, A. Yang, L. Feng and Y. Sun, "Efficient transmission based on RGB LED lamp for indoor visible light communication", Chin. Opt. Lett. 11, 030601 (2013).

[13] O. Gonzalez, R. Perez-Jimenez, S. Rodriguez, J. Rabadan, and A. Ayala, "OFDM over indoor wireless optical channel", in IEE Proceedings of Optoelectronics (2005).

[14] J. Armstrong and A. J. Lowery, "Power efficient optical OFDM", Electron. Lett. 42, 370 (2006).

[15] S.D. Dissanayake, J. Armstrong, "Comparison of ACO-OFDM, DCOOFDM and ADO-OFDM in IM/DD Systems", J. Lightwave Tech. 31, 71063 (2013).

[16] TG7r1, Technical Considerations Document, IEEE 802.15 Std., Rev. 3, https://mentor.ieee.org/802.15/dcn/15/15-15-0492-03-007a-technicalconsiderations-document.docx (July 2015).

[17] J. R. Barry, J. M. Kahn, W. J. Krause, E. A. Lee and D. G. Messerschmitt, "Simulation of Multipath Impulse Response for Indoor Wireless Optical Channels", IEEE J. Sel. Area Comm. 11, 3367 (1993).

[18] F. Lopez-Hernandez, R. Perez-Jimenez, and A. Santamaria, "Modified Monte Carlo Scheme for High-efficiency Simulation of the Impulse Response on Diffuse IR Wireless Indoor Channels", Electron. Letters 34, 191819 (1998). 
[19] F. Lopez-Hernandez, R. Perez-Jimenez, and A. Santamaria, "Raytracing algorithms for fast calculation of the channel impulse response on diffuse IR wireless indoor channels", Opt. Eng. 39, 102775 (2000).

[20] J. Vucic, C. Kottke, S. Nerreter, K. Langer, and J.W. Walewski, "513 Mbit/s Visible Light Communications Link Based on DMT-Modulation of a White LED”, J. Lightwave Tech. 28, 3512 (2010).

[21] R. Li, Y. Wang, C. Tang, Y. Wang, H. Shang and N. Chi, "Improving performance of $750-\mathrm{Mb} / \mathrm{s}$ visible light communication system using adaptive Nyquist windowing”, Chin. Opt. Lett. 11, 080601 (2013).

[22] A. H. Azhar, T. A. Tran and D. O'brien, "A Gigabit/s Indoor Wireless Transmission Using MIMO-OFDM Visible-Light Communications", IEEE Photon. Technol. Lett. 25, 171 (2013).

[23] G. Cossu, R. Corsini, and E. Ciaramella, "High-Speed Bi-directional Optical Wireless System in Non-Directed Line-of-Sight Configuration", J. Lightwave Tech. 32, 2035 (2014).

[24] A. Tsiatmas, C.P.M.J. Baggen, F.M.J. Willems, J.-P.M.G. Linnartz and J.W.M. Bergmans, "An illumination perspective on visible light communications", IEEE Comm. Mag. 52, 764 (2014).

[25] M. Arias, A. Vázquez and J. Sebastián, "An Overview of the AC-DC and DC-DC Converters for LED Lighting Applications", Automatika 53,2156 (2012).
[26] R.O. Caceres and I. Barbi, "A boost DC-AC converter: analysis, design, and experimentation", IEEE Trans. Power. Electron. 14, 1134 (1999).

[27] H. Yuequan and M. M. Jovanovic, "LED Driver With Self- Adaptive Drive Voltage", IEEE Trans. Power. Electron. 23, 3116 (2008)

[28] D. Gacio, J.M. Alonso, A.J. Calleja, J. Garcia and M. Rico-Secades, "A Universal-Input Single-Stage High-Power Factor Power Supply for HBLEDs Based on Integrated Buck-flyback Converter", IEEE Trans. Ind. Electron. 58, 589 (2011).

[29] J.M. Alonso, J. Viña, D.G. Vaquero, G. Martínez and R. Osorio, "Analysis and Design of the Integrated Double Buck-Boost Converter as a High-Power-Factor Driver for Power-LED Lamps", IEEE Trans. Ind. Electron. 59, 41689 (2012).

[30] C. Huang-Jen, L. Yu-Kang, C. Jun-Ting, C. Shih-Jen, L. Chung-Yi and M. Shann-Chyi, "A High-Efficiency Dimmable LED Driver for LowPower Lighting Applications", IEEE Trans. Ind. Electron. 57, 2735 (2010).

[31] H.P.A. van den Boom, W. Li, P.K. van Bennekom, I.T. Monroy, G.-D Khoe, "High-capacity transmission over polymer optical fiber", IEEE J. Sel. Top. Quant. Electron. 7, 3461 (2001).

[32] Y.F. Liu, C.H. Yeh, C.W. Chow, "Alternating-Signal-Biased System Design and Demonstration for Visible Light Communication", IEEE Photonics Journal 5, 7901806 (2013). 